\title{
O USO DAS TICs NO ENSINO MÉDICO: PRIMEIRAS IMPRESSÕES DO PROJETO RESPONDA ${ }^{12}$
}

\author{
Gustavo Emilio Pinheiro Dantas, Centro Universitário de Volta Redonda (UniFOA), \\ gustavopinheiro_@hotmail.com \\ Priscila Almada Torres, Centro Universitário de Volta Redonda (UniFOA), \\ priscilaltorres5@gmail.com \\ Rhanica Evelise Toledo Coutinho, Centro Universitário de Volta Redonda (UniFOA), \\ Universidade Trás-os-Montes e Alto D’ouro (UTAD) \\ profarhanica@gmail.com
}

\begin{abstract}
RESUMO
É perceptível o crescente uso de Tecnologias de Informação e Comunicação (TICs), objeto desse estudo, no meio educacional. O trabalho apresenta uma ação de extensão, realizada no Curso de Medicina do Centro Universitário de Volta Redonda - RJ e tem por propósito utilizar as TICs como metodologia ativa para implementação de um novo meio de comunicação para fins acadêmicos. Propõem-se a criação de um meio de comunicação acadêmico ao mesmo tempo ágil, cômodo e objetivo para a resolução de dúvidas. Toda a plataforma do projeto é virtual, baseada em grupo do () WhatsApp Inc., em que os acadêmicos de diferentes módulos são protocolados e orientados sobre como utilizar a ferramenta para tirar dúvidas. O projeto, que tem período de duração experimental de fevereiro a novembro de 2017, já beneficiou 256 estudantes de todos os módulos do curso, gerando a resolução, até o momento desse resumo, de 123 dúvidas utilizando a plataforma. Esta parceria enriqueceu os meios de interação e trouxe flexibilidade e agilidade aos processos. $O$ uso de Smartphones se mostrou como facilitador de aprendizagem, sendo bem recebido por todos que tiveram acesso aos vídeos e demonstrou ser mais uma estratégia de ensino para facilitar e despertar o interesse na aprendizagem.
\end{abstract}

PALAVRAS-CHAVE: Ensino Médico; TICs; Redes Sociais.

\section{“RESPONDA PROJECT”: THE USE OF ICT'S IN THE MEDICAL ACADEMIC ENVIRONMENT AND IT'S FIRST IMPRESSIONS}

\begin{abstract}
The increasing use of Information and Communication Technologies (ICTs) in education is notorious. This partnership enriched the means of interaction, in addition to bringing flexibility and agility to the processes related to it. The purpose of this extension work, carried out at the University Center of Volta Redonda - RJ, is to use ICTs as a methodology for the

\footnotetext{
${ }^{1}$ O presente trabalho contou com apoio financeiro do UniFOA para sua realização.

${ }^{2}$ Produto do Laboratório do Grupo de Estudos e Pesquisas em Representações Sociais na/para Formação de Professores - LAGERES/CNPq.
} 
implementation of a new environment for academic purposes. The objective of the work is to create a tool of academic communication that is at the same time agile, comfortable and objective for solving doubts. The entire project platform is virtual, group-based on (C) WhatsApp Inc., where students from different modules and medical education levels are logged and guided on how to use the tool for questions. The project, which has an experimental period of February to November 2017, has already benefited 256 students from all levels and stages of the medical course, providing the resolution up to the moment of this summary of 123 doubts using the platform. We noticed that the use of ICTs was an enabler of learning and was well received by all who had access to the videos and proved to be another weapon to facilitate interests and turn learning an easier process. Here it is the appropriate scenario to insert your abstract. This text cannot exceed the limit of 500 characters (spaces included). The abstract must be informative and it must include objective, methodology and results.

Key Words: Health Sciences, Technology And Communication, TICS.

\section{PROYECTO RESPONDA: USO DE LAS TICES EN MEDIO MÉDICO ACADÉMICO Y SUS PRIMERAS IMPRESIONES}

\section{RESUMEN}

Es perceptible el creciente uso de Tecnologías de Información y Comunicación (TICs) en el medio educativo. Esta asociación enriqueció los medios de interacción, además de traer flexibilidad y agilidad a los procesos a ella relacionados. El presente trabajo de extensión, realizado en el Centro Universitario de Volta Redonda - RJ, tiene por propósito utilizar las TIC como metodología para la implementación de un nuevo medio de comunicación para fines académicos. El objetivo del trabajo es crear un medio de comunicación académico al mismo tiempo ágil, cómodo y objetivo para la resolución de dudas. Toda la plataforma del proyecto es virtual, basada en grupos de (C) (C) WhatsApp Inc. Inc. donde los alumnos de diferentes módulos son protocolados y orientados sobre cómo utilizar la herramienta para dudar. El proyecto que tiene un período de duración experimental de febrero a noviembre de 2017, ya benefició a 256 estudiantes de todos los períodos del curso de Medicina, generando la resolución, hasta el momento de ese resumen de 123 consultas utilizando la plataforma. Se observa que el uso de TIC se mostró como un facilitador de aprendizaje, siendo bien recibido por todos los que tuvieron acceso a los vídeos y demostró ser más un arma para facilitar el interés y la facilidad en el aprendizaje.

Palabras Clave: Ciencias De La Salud, Tecnología Y Comunicación, Tics.

\section{INTRODUÇÃO}

As Tecnologias da informação e Comunicação (TICs), cerne do estudo, estão cada vez mais presentes no cotidiano da população. A informática não é mais um privilégio de poucos; mas sim a necessidade de muitos. O mundo está sendo movido por novas tecnologias e não há 
como negar as possibilidades de transformação da cultura e da educação nos dias atuais (MANGAN, 2010). Nesse sentido, o Projeto Interdisciplinar TICs Medicina, desenvolvido no curso de Medicina do Centro Universitário de Volta Redonda - UniFOA, com apenas dois anos de existência, tornou-se um celeiro para desenvolvimento de ideias, projetos, produtos e produção científica voltados para o uso das TICs.

Entendemos que o conceito de sociedade das comunicações móveis é remoto (CASTELLS, 2003); no entanto, torna-se essencial inserir esse espaço de comunicação rápida e dinâmica no contexto do ensino médico, uma vez que estamos envoltos a uma cultura hipertecnológica (SERROY; LIPOVETSKY, 2011). Dessa forma, o que há de novo e inédito com as tecnologias da informação e da comunicação é a parceria cognitiva que elas estão exercendo na relação que o aprendente estabelece com elas (ASSMANN, 2000).

Pensando nisso, percebemos que o futuro da comunicação interpessoal caminha de mãos dadas com ferramentas que facilitem o modo como interagimos com o outro e que caminhe em uma direção que seja benéfica para ambas as partes. No cotidiano da população, o uso da ferramenta (C) WhatsApp Inc., por meio de dispositivo móvel (mobile) é difundido por todos, possuindo um bom know-how de usuários ativos diariamente.

Nesse sentindo, indagamos: O uso de dispositivo móvel por meio do aplicativo (C) WhatsApp Inc. pode ser adaptado como ambiente de ensino aprendizagem entre acadêmicos do Curso de Medicina? E nesse caso, quais os processos e estratégias mais adequados para a troca de conhecimento entre estudantes?

Para encontrar respostas as nossas questões, criamos como um dos produtos do Projeto TICs Medicina, uma ação de extensão denominada "Projeto Responda" que tem por objetivo criar uma rede de comunicação interativa em que os acadêmicos de módulos distintos possam dialogar de forma prática entre si, sanar dúvidas, ajudando e cooperando mutuamente a aprenderem em comunidade acadêmica; mapear dados relacionados às dúvidas mais frequentes dos estudantes, entre outros, em somatório. Nesse sentido, o estudo visa a apresentar esse relato de experiencia.

Acreditamos que a ação de extensão proposta venha auxiliar os estudantes do curso de Medicina do UniFOA, agilizando a resolução de dúvidas, assim como também auxiliando os acadêmicos que estão prestes a concluir a graduação a relembrarem conteúdos, que futuramente serão cobrados na seleção para residência médica. 
O processo de ensino-aprendizagem necessita que duvidas existam para ser bemsucedido. Isso faz parte da complexa dinâmica do ensino. Sendo assim, a justificativa do então intitulado "Projeto Responda" é ser um meio facilitador de dúvidas, ou seja, seu objetivo é sanar dúvidas de forma rápida e segura, encurtando distâncias entre quem possui indagações e alguém que detenha o conhecimento para saná-la. Além disso, o trabalho se justifica por atender uma das necessidades preconizadas pelas novas DCNs (2014) do Curso de Medicina, que determinam o uso das TICs no ensino.

Dessa forma, proporcionamos, por meio tecnológico, uma aproximação entre alunos de módulos distintos; utilizando essa ferramenta tecnológica de código aberto para todos, possibilitando que os alunos tenham um espaço voltado exclusivamente para auxiliar seu aprendizado, com maior agilidade.

\section{METODOLOGIA}

Trata-se de um relato de experiência referente ao projeto de extensão desenvolvido no Curso de Medicina do UniFOA, desenvolvido por meio de pesquisa de natureza mista do tipo exploratória (CRESWELL, 2010) e de levantamento bibliográfico. O estudo foi ancorado nas Dimensões da Pesquisa Propostas por Novikoff (2010, p.222), que para a autora: “independente da abordagem da pesquisa (CRESWEL, 2007) passam por cinco etapas, didaticamente organizadas, para melhor orientar o ensino-aprendizagem da pesquisa desde o desenvolvimento e na apresentação escrita das mesmas”.

As Dimensões da Pesquisa Propostas por Novikoff (2010, p.6) são consideradas como: "abordagem teórico-metodológica, com todas as dimensões de preparação, estudo, desenvolvimento e apresentação de pesquisa acadêmico-científica", constituídas com objetivo de organizar o pensamento científico.

Como primeira etapa, desenvolvemos a dimensão epistemológica, em que buscamos identificar e delimitar o objeto de estudo, assim como os objetivos, a problematização, a hipótese e a justificativa. A seguir, delineia-se a dimensão teórica - espaço de ancoragem dos conceitos basilares do estudo - ou seja, Ensino Médico, TICs e Mídias Sociais. Em sequência, a dimensão técnica traz a apresentação do caminho metodológico adequado à proposta. A dimensão morfológica, quarta a ser desenvolvida, se estabelece como base para a 
apresentação de dados e resultados. Como última, a dimensão analítica-conclusiva, traz as considerações finais da pesquisa.

Ainda no contexto metodológico, buscamos compreender o que os teóricos discutem sobre TICs, ensino aprendizagem por meio mobile ou aparelhos móveis e redes sociais. Em um segundo momento, reunimos informações e criamos um grupo que integralizasse estudantes de módulos distintos na rede. Assim, foram criados instrumentos ou aparatos ou apetrechos para padronizar a metodologia de utilização das ferramentas voltadas para a aprendizagem e resolutividade de duvidas acadêmicas que caminham de mãos dadas com o processo de ensino-aprendizagem.

No contexto prático o "Projeto Responda" possui padrões de perguntas e respostas estipulado: ao perguntar, o participante deverá incluir na mensagem o seu nome, sua matrícula, sobre qual disciplina é a sua dúvida, juntamente a um breve tema. Por exemplo: "Meu nome é Maria Fernandes, matrícula 201820014. Tenho uma dúvida de Fisiologia, sobre fisiologia cardíaca.”. Já o aluno que se dispuser a responder à pergunta, deverá seguir o mesmo padrão de nome e matrícula, acrescentando que responderá à pergunta em questão. Posteriormente, os alunos seguem para uma conversa privada em cuja qual a dúvida será esclarecida e deverá ser comentado no grupo que a dúvida foi sanada, caso isso tenha acontecido. Essa é uma maneira de não poluir o grupo visualmente e evitar que as perguntas se percam em meio às discussões sobre alguma resposta. Além disso, é um método que visa à coleta de dados, feita durante o semestre para construir as estatísticas descritas no artigo.

Como resultado, a proposta prevê, ainda, render horas de atividades extracurriculares para os alunos voluntários, gerando um benefício mútuo, e servir como atrativo para adesão de membros interessados. Em outra esteira, o projeto propõe o levantamento de dados por meio de pesquisa, pretendendo identificar e mapear as incidências de dúvidas e, consequentemente, gerar um relatório que será compartilhado com os supervisores de módulos. A partir desses dados, as lideranças poderão identificar quais são as maiores dúvidas elencadas pelos participantes e, por conseguinte, compartilhar com a equipe docente.

Em outra perspectiva, a proposta auxiliará os acadêmicos voluntários dos módulos finais, que tem a necessidade de rever os conteúdos estudados nos anos iniciais, visto que esses são cobrados em provas para a residência; dessa forma, a proposta propicia a oportunidade de reciclagem constante, porém vinculada a uma ação solidária. 


\section{APRESENTAÇÃO DE DADOS E RESULTADOS DO PROJETO}

As TICs, como ferramentas didáticas, por meio do uso da internet, ampliam as possibilidades da prática educacional e pedagógica, ou seja, "a utilização dessas tecnologias digitais também nos permite a atualização de conhecimentos, a socialização de experiências e aprendizagem permanente através do desenvolvimento de trabalhos cooperativos" (RABELO MORAES; ESCOLA, 2014, p. 281); são utilizadas na educação em contextos muito diferenciados, com objetivos e formas de exploração distintas. A situação mais comum é talvez a sua utilização em sala de aula, como suporte às atividades de ensino.

O ensino médico preconiza a partir das Diretrizes Nacionais Curriculares (DCNs, 2014) o uso das TICs, o que viabiliza uma maior aproximação dos estudantes acerca do uso de estratégias inovadoras, que poderão auxiliá-lo futuramente no mercado de trabalho. Nesse contexto, para Almeida, Raimundo e Escola (2014, p. 317)

Tendo as TIC um impacto tão decisivo nos cuidados de saúde, o seu uso no ensino dos futuros profissionais, não pode ser descurado. Conseguir uma boa integração deste fenómeno nos processos ensino-aprendizagem pode ser uma mais-valia na sua formação. Desde logo, porque se o serviço de saúde aposta nesses recursos, o futuro profissional deve estar preparado para o uso das diferentes ferramentas existentes de modo a saber retirar de cada uma o melhor rendimento possível de forma a desenvolver competências técnicas e relacionais para uma assistência individualizada aos utentes.

Nessa linha, outra vertente de utilização das TICs está associada aos espaços e momentos de autoestudo, recorrendo a documentos em suportes digitais (GOMES; PICH; VAZ, 2006), assim como também às comunidades virtuais que se estabelecem em prol da aprendizagem. As redes são estabelecidas por sujeitos que possuem afinidades, que funcionam como conectores (MATTAR, 2013), variando a interação de acordo com a disponibilidade de tempo e espaço para o diálogo, independente da distância geográfica.

Esse formato de interação por meio de redes sociais adequa-se perfeitamente ao "Projeto Responda", baseado na ajuda de aluno para aluno. Trata-se de um grupo criado na plataforma (C) WhatsApp Inc., que inclui alunos de todos os módulos do curso de medicina do Centro Universitário de Volta Redonda, com o intuito de tirar as dúvidas por pessoas da mesma turma e/ou de turmas diferentes, de maneira cômoda e, principalmente, ágil. A plataforma escolhida foi o (C) WhatsApp Inc., por possuir exatamente essas características; 
haja vista que é um produto de mensagens instantâneas e que a maioria das pessoas já possui e usa com frequência.

O Projeto é amplamente educativo, pois não é só quem tem suas dúvidas sanadas que aprende. $\mathrm{O}$ aluno que responde às perguntas também consolida seu conhecimento, favorecendo, assim, ambos os lados e ainda pode, por meio dessa ação, relembrar conteúdos que o auxiliaram sempre.

\subsection{Material de Apoio}

Foi criada a marca do "Projeto Responda" e a identidade visual para o material promocional, intencionando fortalecer a imagem do projeto e, desse modo, captar novos participantes.

\subsection{Resultados Parciais}

Como resultados parciais, pretendemos exemplificar o que o projeto já conquistou até a data do presente resumo, demonstrando numericamente como o "Projeto Responda" se tornou um meio útil para a aprendizagem dos alunos. Nesse sentido, obtivemos 123 perguntas efetuadas durante o semestre letivo que compreende 01 de fevereiro de 2017 até 11 de maio de 2017.

Com uma representatividade de aproximadamente $1 / 3$ de todos os alunos do curso de Medicina do Centro Universitário de Volta Redonda - RJ, o projeto serviu como um excelente agregador de dúvidas e apresentou uma resolutividade que ultrapassou o que esperávamos inicialmente. Com a ótima adesão dos alunos, o projeto foi sofrendo aprimoramentos durante seu curso, mas pretendemos trazer alguns resultados importantes, que justifiquem sua qualidade e também como pretendemos melhorá-lo em diante. 


\subsection{Matérias Mais Questionadas}

Participando do "Projeto Responda", alunos desde o primeiro módulo do curso de medicina até alunos do 12 módulo. Isso fez com que as dúvidas variassem entre matérias do ciclo básico, ciclo clínico e internato. O gráfico 01 exemplifica todas as matérias pesquisadas no período de fevereiro a maio de 2017 e sua respectiva incidência de perguntas.

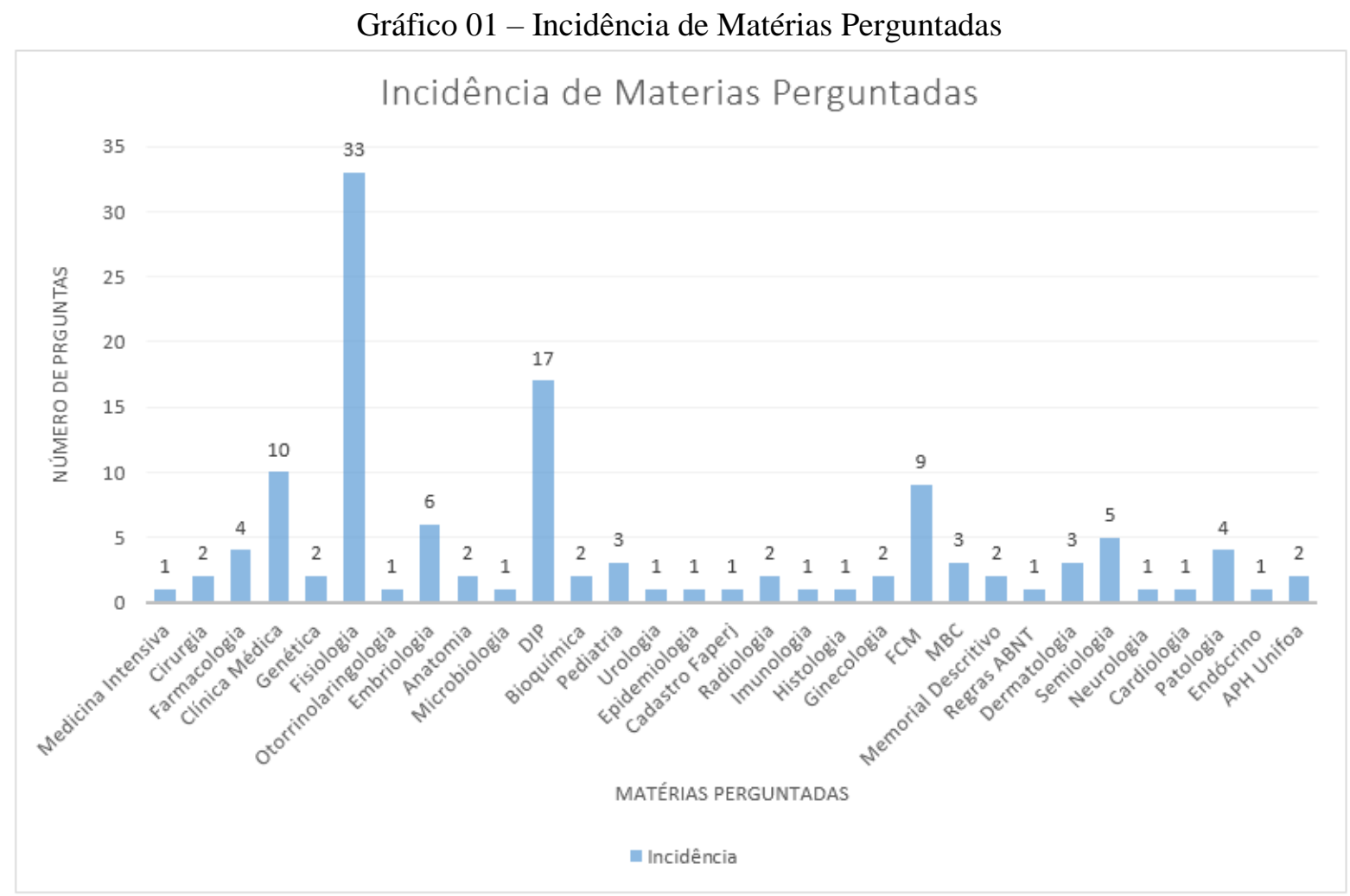

Fonte: Elaborado pelos autores

O mapeamento dos dados será desenvolvido no decorrer dessa ação de extensão, que termina em novembro de 2017 - sua segunda fase - em que poderemos, assim, extrair informações que trarão novas ideias e que poderão nortear a evolução dessa proposta. 


\section{CONSIDERAÇÕES FINAIS}

Como o projeto de extensão, desenvolvido a partir do dispositivo móvel por meio do aplicativo (C) WhatsApp Inc., se mostrou extremamente funcional e ajudou muitos alunos; a proposta evoluiu, atraindo mais interessados e, além disso, com a adesão de uma nova turma ao projeto, foram necessários alguns ajustes e mudanças. A ideia é que todos os interessados tenham seu espaço no projeto, e que, no futuro, ele possa ser utilizado por todos os alunos, e quem sabe até esse modelo possa ser utilizado por outras Instituições de Ensino.

Para ampliar o alcance, será criado mais um grupo no aplicativo (c) WhatsApp Inc., para o qual metade dos alunos já presentes no projeto serão transferidos, buscando a maior igualdade possível na distribuição. Com isso, haverá vagas suficientes para os alunos da turma que ingressou na faculdade esse semestre (2017.2) e, para os alunos que demonstrarem interesse depois do começo das atividades, quando o grupo inicial já estiver lotado. Os dois grupos funcionarão da mesma maneira, descrita anteriormente, e não será alterada com base nas opiniões dos participantes nas pesquisas feitas ao final do semestre 2017.1.

Entretanto, houve citações, algumas vezes, sobre a vontade de conhecer as perguntas de outros alunos. Para isso, foi encontrada uma maneira de permitir que os participantes conheçam as outras dúvidas, sem que o método inicial fosse muito modificado. Basicamente, será estipulado que o participante, que estiver interessado na dúvida de outra pessoa, mande um caractere especial determinado e, assim, o autor da pergunta deverá mandar sua pergunta e a resposta para essa pessoa.

A expectativa é que essas mudanças tragam mais atenção e visibilidade para o projeto, ampliando-o cada vez mais. Dessa forma, no próximo semestre (2018.1), pretende-se propor a integração do "Projeto Responda" ao programa de monitoria do Curso de Medicina, incluindo nos grupos todos os monitores de todas as disciplinas, mas sem que eles se tornem os únicos responsáveis pelas respostas dos outros participantes. Este estudo não se esgota aqui, uma vez que, ao findar o cronograma de ações proposto, serão contabilizadas as pesquisas desenvolvidas no decorrer dessa ação de extensão; deixamos, assim, novas indagações referentes aos tipos de dúvidas, tempo de respostas, dentre outras.

\section{REFERÊNCIAS BIBLIOGRÁFICAS}


ALMEIDA, C.; F. M. RAIMUNDO; ESCOLA, José Jacinto Joaquim. Tecnologias de informação e comunicação nos cuidas de saúde: um portal ou um muro comunicacional? In: ESCOLA, Joaquim José Jacinto et all. Rumo à inclusão educacional e integração das TIC na sala de aula. Santiago de Compostela, Espanha: Andarivas Editores, 2014.

ASSMANN, H. A metamorfose do aprender na sociedade da informação. Ci. Inf., v. 29, n. 2, 2000.

BRASIL. Ministério da Educação. Conselho Nacional de Educação. Câmara de Educação Superior. Resolução No 3, de 20 de junho de 2014. Institui Diretrizes Curriculares Nacionais do Curso de Graduação em Medicina. Diário Oficial da União. Brasília,2014.

CASTELLS, M. A Galáxia Internet: reflexões sobre a Internet, negócios e a sociedade. Zahar, 2003.

GOMES, I. M.; PICH, S; VAZ, A. F. Sobre algumas vicissitudes da noção de saúde na sociedade dos consumidores. Revista Brasileira de Ciências do Esporte, Campinas, v. 27, n. 3, p. 137-151, maio 2006.

MANGAN, P. K. V., SARMENTO, D. F., MANTOVANI, A. M. As Tecnologias da Informação e da Comunicação: recortes de experiências no contexto da formação inicial do professor. Colabor@ - Revista Digital da CVA - Ricesu, v. 6, n. 22, 2010.

MATTAR, J. Web 2.0 e redes sociais na educação. São Paulo: Artesanato Educacional, 2013.

RABELO MORAES, N. F.; ESCOLA, J. J. J. Tão longe mas tão perto: redes sociais espaço de aprendizagem digital cooperativo. In: ESCOLA, Joaquim José Jacinto et all. 
Rumo à inclusão educacional e integração das TIC na sala de aula. Santiago de Compostela, Espanha: Andarivas Editores, 2014.

SERROY, J.; LIPOVETSKY, G. A cultura-mundo: resposta a uma sociedade desorientada. São Paulo: Companhia das Letras, 2011. 